Corrigendum

\title{
Corrigendum to: "Vaccination in immunocompromised host: Recommendations of Italian Primary Immunodeficiency Network Centers (IPINET)" [Vaccine 36 (2018) Pages 3541-3542]
}

\author{
Baldassarre Martire ${ }^{\mathrm{a}, *}$, Chiara Azzari ${ }^{\mathrm{b}}$, Raffaele Badolato ${ }^{\mathrm{c}}$, Clementina Canessa ${ }^{\mathrm{b}}$, \\ Emilia Cirillo ${ }^{\mathrm{d}}$, Vera Gallo ${ }^{\mathrm{d}}$, Simona Graziani ${ }^{\mathrm{e}}$, Tiziana Lorenzini ${ }^{\mathrm{c}}$, Cinzia Milito ${ }^{\mathrm{f}}$, \\ Raffaella Panza $^{a}$, Viviana Moschese ${ }^{e}$, with Italian Network for Primary Immunodeficiencies (IPINET) ${ }^{1}$ \\ a Pediatric Hematology Oncology Unit, "Policlinico-Giovanni XXII" Hospital, University of Bari, Italy \\ ${ }^{\mathrm{b}}$ Pediatric Immunology Unit "Anna Meyer" Hospital University of Florence, Italy \\ ${ }^{\mathrm{c}}$ Department of Clinical and Experimental Sciences, University of Brescia, Italy \\ ${ }^{\mathrm{d}}$ Department of Translational Medical Sciences, Pediatric section, Federico II University, Naples, Italy \\ e Pediatric Allergology and Immunology Unit, Policlinico Tor Vergata, University of Rome Tor, Vergata, Italy \\ ${ }^{\mathrm{f}}$ Department of Molecular Medicine, Sapienza University of Rome, Italy
}

The authors regret that the names of some of the centers that

participated in the study were omitted from the original

publication: the full list of the IPINET members and centers is given above.

\footnotetext{
DOI of original article: https://doi.org/10.1016/j.vaccine.2018.01.061

* Corresponding author.

E-mail address: baldo.martire@gmail.com (B. Martire).

${ }^{1}$ Italian Primary Immunodeficiencies Network (IPINet) is: Coordinator: C. Pignata, Department of Translational Medical Sciences, Pediatric section, Federico II University, Naples.

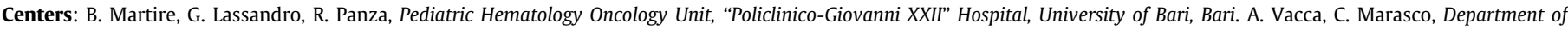

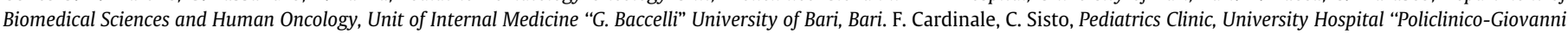

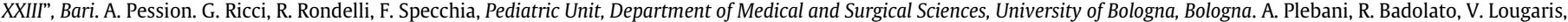

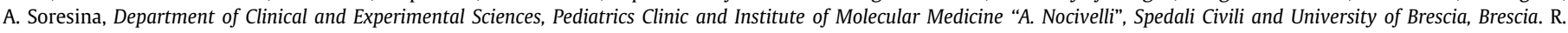

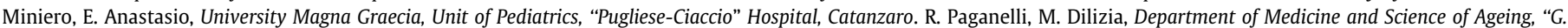

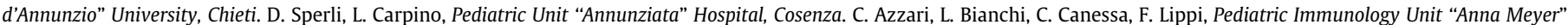

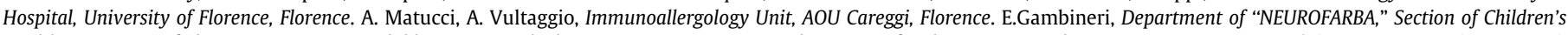

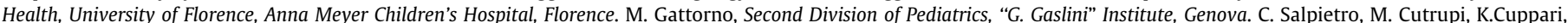

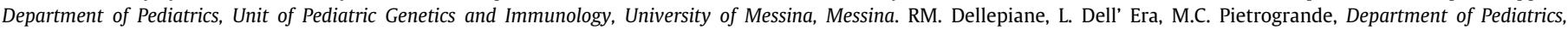

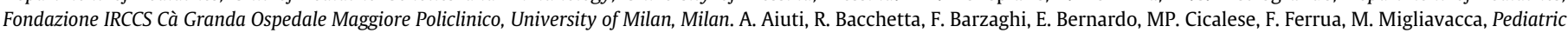

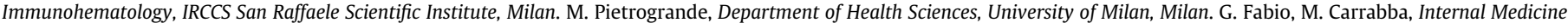

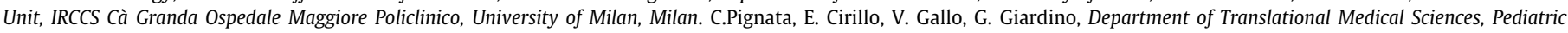

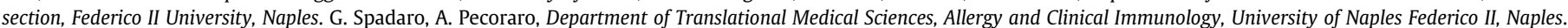

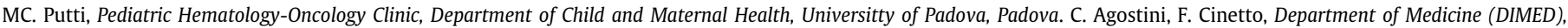

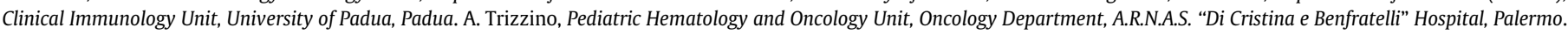

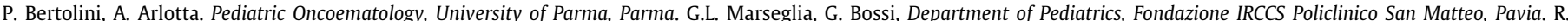

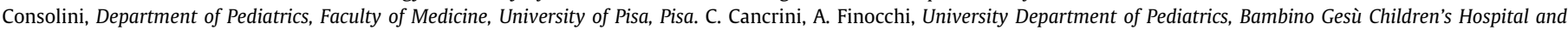

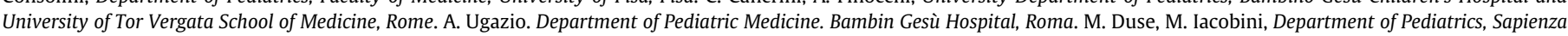

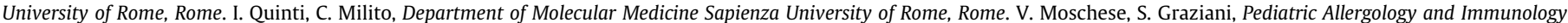

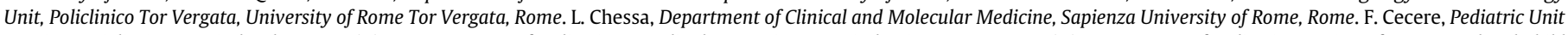

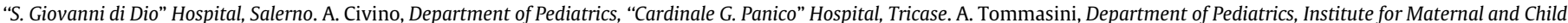

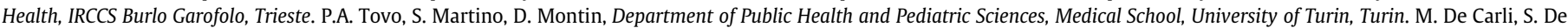

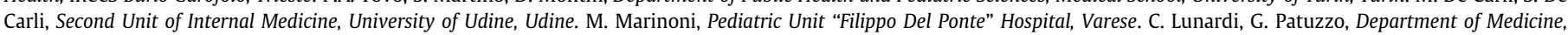
University of Verona, Verona.
} 